\title{
NIVELES DE ALFABETIZACIÓN \\ EN LA ESPAÑ̃ DEL SIGLO XVIII \\ Primeros resultados de una encuesta en curso
}

\author{
Por Jacques SOUBEYROUX \\ Universidad de Montpellier
}

Este artículo se propone presentar el método puesto a punto y los primeros resultados alcanzados en una encuesta en curso sobre la alfabetización en la España del XVIII, que forma parte de un programa de investigación más amplio sobre la sociedad española del siglo XVIII, que dirijo en la Universidad de Montpellier desde 1979, y que vamos desarrollando a partir de encuestas llevadas a cabo en diferentes provincias de España, sobre todo en los archivos de protocolos. Estas encuestas tratan de estudiar conjuntamente los niveles de riqueza a partir de los inventarios de bienes, y los niveles de alfabetización y cultura, cuyas fuentes principales son los testamentos.

La alfabetización constituye un campo de investigación inmenso que procuramos abordar a tres niveles complementarios:

-el problema de la educación, en particular en la primera enseñanza,

- la medida de la alfabetización, o sea la proporción de los que eran capaces de leer y escribir en la España del XVIII, y quiénes eran,

-la difusión del libro y la lectura.

De esos tres niveles, sólo desarrollaré aquí el segundo, por ser en el que hemos adelantado más en nuestra investigación (me ha parecido que, al alcanzar la etapa de las diez mil firmas, se podía hacer un primer balance), y quizás el menos explorado aún en España. (1) Sobre el primero me contentaré con algunas palabras que me servirán para introducir el tema de los niveles de alfabetización.

El libro de R. KAGAN, Universidad y sociedad en la España moderna (Madrid, 1981), dedicado sobre todo a los siglos XVII y XVIII, señala en su capítulo primero (Enseñanza de las primeras letras) direcciones a explorar que siguen vigentes para el XVIII: 
- la familia y el hogar, espacio muy mal conocido aun,

- las escuelas de primeras letras.

Sobre este último punto, los censos del XVIII aportan datos interesantes: el Catastro de La Ensenada permite hacer un inventario de los pueblos del reino de Castilla que tenían ya maestros de primeras letras a mediados del siglo.(2) Para completar, es posible acudir a las fuentes asistenciales y a los archivos de las Sociedades Económicas de Amigos del País, que encierran documentos sobre las escuelas pías o gratuitas, y las escuelas patrioticas, fundadas en muchas ciudades durante los últimos veinte años del siglo. Por ejemplo, las Diputaciones de Barrio de Madrid y la Sociedad Económica Matritense crearon escuelas gratuitas en casi todos los barrios de la Corte, que acogían en 1799 a 1.608 niñas, que recibían una enseñanza práctica, y a 843 niños, que, además de la doctrina, aprendían a leer, escribir y sumar. En estas escuelas, los métodos pedagógicos seguían los mismos que en los dos siglos anteriores: en particular, el aprendizaje de la lectura precedía al de la escritura. ${ }^{(3)}$

La red de escuelas de primeras letras permite conocer las posibilidades existentes en la España del XVIII para aprender a leer y escribir. La etapa siguiente consistirá en medir la alfabetización.

La medida de la alfabetización es un problema complejo porque ignoramos las bases de alfabetización en la época anterior (siglos XVI y XVII), es decir los principios de la alfabetización en la época moderna. Las únicas cifras que poseemos son la tasa de analfabetismo en el Censo de 1900: un 56,07\% de analfabetos (un 45,58 de hombres y un 65,89 de mujeres) y la evaluación de LUZURIAGA para los años de 1870: un 75\% de analfabetos. (4) $^{(4)}$

El retraso de la alfabetización en España en 1900 se debe,

- ¿A un arranque más tardío? La cosa parece improbable: La España del XVI no está menos alfabetizada que los demás países europeos.

- ¿A un crecimiento más lento y más difícil? Más probablemente. Pero este crecimiento ifue continuo, regular (como en el siglo XX), o por lo contrario, pasó por fases de aceleración y por pausas, hasta regresiones? Esta última hipótesis me parece la más verosímil, y la segunda mitad del XVIII, marcada por la extensión de la red de escuelas de primeras letras, puede ser una de esas fases de aceleración del proceso: es una de las hipótesis que nuestras encuestas tratarán de comprobar.

\section{Problemas metodológicos}

¿Cómo conocer el número de analfabetos en la España del XVIII? Lo más sencillo es mirar lo que se hizo en los países vecinos, por ejemplo en Francia donde la investigación sobre niveles de alfabetización está bastante adelantada. Esta investigación empezó desde los años de 1877-1879 con la encuesta del Rector MAGGIOLO, constituida por cinco sondeos retrospectivos hechos gratuitamente por maestros en los archivos parroquiales (libros de casamientos) y municipales, y relativos a los años de 1686-1690, 1786-1790, 1816-1820, 1866 y 1872-1876. Esta encuesta ha sido criticada en sus métodos y su realización práctica, pero sus resultados están ampliamente confirmados por las investigaciones recientes: un 
$79 \%$ de analfabetos a fines del XVII, que se reducen a un $63 \%$ a fines del XVIII, con una Francia del Norte (línea Saint-Malo-Ginebra) más alfabetizada (todos los departamentos excepto uno tienen más de un $30 \%$ de alfabetizados) que la Francia del Sur (todos los departamentos menos 4 tienen menos del $30 \%$ de alfabetizados).

El método utilizado es el test de la firma, o sea la capacidad para los novios (hombre y mujer) de firmar el libro de casamiento. Pero ¿hasta qué punto poder firmar significa saber leer y escribir? Ante esa equivalencia, los historiadores franceses han adoptado tres posiciones principales:(5)

- la tesis hipercrítica de Yves CASTAN, para quien la firma no es más que «el grado cero de la escritura», y es independiente de la aptitud para leer y escribir. Más próxima al signo que al escrito, la firma aprendida y dibujada letra por letra (muchas veces con la ayuda de un modelo) sólo traduce una voluntad de afirmación social. Esta teoría me parece pertinente en ciertos puntos (la firma como afirmación social), pero lo que dice CASTAN sólo vale para ciertas firmas, y no puede generalizarse.

- la tesis media de Jean MEYER quien, a partir de la separación operada por la pedagogía antigua, entre lectura y escritura, afirma que saber firmar constituye un nivel intermedio entre los dos aprendizajes. El grupo de los que firman reuniría así a los que aprendieron a leer y tienen algunas nociones de escritura, y a los que habían aprendido a escribir y han perdido, por falta de práctica, el dominio de esa técnica. Paradójicamente, saber firmar significaría saber leer, y no siempre saber escribir.

- la tesis favorable de François FURET, quien comparando tres fuentes correspondientes al mismo año de 1866: las firmas de los libros de casamientos (encuesta MAGGIOLO).

Los datos del primer censo de la población francesa, en que aparecen especificados los que saben leer y escribir, los que sólo saben leer, y los que no saben leer ni escribir.

Los datos del reclutamiento militar en que figuran las mismas categorías, muestra, a partir de un análisis factorial, la estrecha correlación (coeficiente 0,91 ) que existe entre saber firmar (encuesta MAGGIOLO) y alfabetización completa (los que saben leer y escribir en el censo y en los registros militares). En cambio, no existe ninguna correlación entre saber firmar y saber leer sólo. La firma es así el revelador de una alfabetización completa.

Lo que vale para 1866, en una época en que la pedagogía ha evolucionado y en que está abandonada ya la antigua separación entre aprendizaje de la escritura y de la lectura (pero no pocas personas que figuran en el censo de 1866 habrán conocido todavía el antiguo sistema pedagógico), ¿vale también para el siglo precedente? Ninguna fuente permite afirmarlo, pero existen fuertes probabilidades. Además, como subraya F. FURET, los mismos resultados de la encuesta de MAGGIOLO, por la masa de documentos tratados (220.000 para fines del XVII, 344.000 para fines del XVIII) tienen una coherencia que no existiría si la firma no tuviera ninguna significación, como pretende Yves CASTAN.

La firma es sin duda un acto individual, la afirmación de una identidad, pero es un acto relacionado con todo un entorno social (progresos del sistema 
educativo, presión del modelo administrativo, etc...), que tiene pues una significación social: firmar un testamento, una carta de dote, una declaración jurada, es participar de unos valores sociales. La firma no es un signo arbitrario, sino el revelador de todo un contenido social. por eso es pertinente considerarla como objeto de estudio.

La firma, sí. Pero, ¿qué documentos utilizar? En la Francia del Antiguo Régimen, los libros parroquiales de matrimonios firmados por los novios constituyen una fuente privilegiada, de fácil utilización y de excelente calidad, representativa de todas las clases sociales. El problema es que, en España, salvo raras excepciones en el siglo XVI, estos libros están firmados por el cura párroco, y no por los novios. De ahí la necesidad de acudir a otras fuentes: los protocolos, los testamentos en particular (utilizados también en Francia por Michel VOVELLE en Provenza y Daniel ROCHE en París), las fuentes fiscales e inquisitoriales.

A - Fuentes fiscales: las más utilizadas hasta aquí han sido los «donativos», impuesto extraordinario pagado con motivo de una guerra, para el cual cada vecino tenía que presentar una declaración jurada de sus propiedades y rentas, y firmar cuando sabía. La ventaja del documento consiste en poder comparar en cada caso la capacidad contributiva del otorgante con su capacidad para firmar. El principal inconveniente es que varias categorías están excluidas. Así, en el caso del donativo de 1635 estudiado en Santiago de Compostela por Juan Eloy GELABERT ${ }^{(6)}$, sólo 984 vecinos de un total de 1.490 figuran en las deciaraciones juradas. El $28 \%$ de esos 984 vecinos sabe firmar: concluir que, añadiendo al clero (que se supone alfabetizado en su totalidad) la mitad de la población de Santiago sabía firmar, me parece una inducción peligrosa.

Este tipo de documentos existe para el siglo XVIII (donativos de 1705, 1771, 1797 , etc.) y la primera mitad del XIX, y las relaciones juradas correspondientes están conservadas en los archivos municipales. ${ }^{(7)}$

$B$ - Fuentes inquisitoriales: las firmas de los acusados y testigos de los procesos de los tribunales inquisitoriales de Toledo y Córdoba han sido estudiados por Marie-Christine RODRIGUEZ y Bartolomé BENNASSAR. ${ }^{(8)}$ Los resultados son eufóricos: un 75\% de firmantes para el periodo de 1751-1817 (el 91\% de los hombres y el $14 \%$ de las mujeres saben firmar), o sea una tasa de alfabetización muy superior a la alfabetización francesa de la misma época y a la tasa española de 1900 ( $56 \%$ de analfabetos). Esa cifra me parece altamente improbable, y obliga a considerar que la fuente misma que se utiliza para el siglo XVIII no es representativa de la sociedad de la época.

C - Fuentes notariales: el mejor ejemplo de su utilización hasta la fecha me parece ser el artículo de Claude LARQUIE sobre «La alfabetización de los madrileños en $1650 » .{ }^{(9)} \mathrm{A}$ partir de 1413 testamentos y declaraciones de pobres (que representan más del $1 \%$ de la población de Madrid en la época), LARQUIE llega a una tasa de un $39,2 \%$ de firmantes, a los que añade los casos de los que no han podido firmar «por la gravedad de su enfermedad», para concluir que el 45,35\% de los madrileños sabían firmar, contra el 54,6 que no sabían.

Nuestra investigación, iniciada en $1979,{ }^{(10)}$ se funda esencialmente en los tes- 
tamentos por ser éste un documento masivo, que debía estar firmado para ser válido. Su utilización no deja sin embargo de plantear un problema de representatividad sociológica de la muestra de pcblación así alcanzada: el testamento es un documento que traduce preocupaciones a la vez sociales y espirituales (organización de la ceremonia del entierro, número de misas, etc...) que corresponden más bien a una población urbana, mientras los inventarios de bienes son en su mayoría rurales. El peligro evidente es el de una sobrerrepresentación de las clases privilegiadas urbanas, las más alfabetizadas, por lo que nuestros resultados tenderán a pecar por exceso de optimismo. Este peligro es el de casi todas las fuentes cuando se trata de alfabetización: a pesar del recurso tan frecuente al notario en los países mediterráneos, no cabe duda que las clases inferiores no están debidamente representadas en las actas testamentarias.

Este defecto está atenuado en Madrid, donde existen declaraciones de pobres, documento que substituye al testamento con el mismo objeto para los que, no teniendo ningún bien, piden que los entierren de limosna. ${ }^{(1)}$ Pero, en general, nuestra muestra tendrá un nivel sociocultural superior a la media, elemento que será necesario tener en cuenta en el momento de apreciar los resultados.

Uno de los requisitos del método consiste en estudiar todos los protocolos de un mismo año en una misma ciudad o provincia. Cada escribano tenía, en efecto, una clientela dotada de rasgos particulares, y contentarse con unos sondeos podría falsear completamente los resultados. Esta condición es fácil de realizar en ciudades pequeñas o medias, como Burgos (16 escribanos a mediados del XVIII) o Murcia (38 escribanos). Impone una labor más exigente para Madrid (224 escribanos a fines del XVIII).

Los datos que figuran en los documentos notariales son los siguientes:

-nombre y apellido,

- lugar de residencia,

- lugar de nacimiento del otorgante,

-nombres, apellidos y lugares de nacimiento de sus padres, ${ }^{(12)}$

- estado civil, con el nombre y apellido del cónyuge,

- no siempre, calidad o profesión,

- firma (o no firma) con especificación de los diferentes casos por el escribano:

- «lo firmó»,

- «no lo firmó por no saber»,

— «no lo firmó por no poder» (o «por la gravedad de su enfermedad», «por la convulsión que padece», etc...).

Los dos primeros casos no plantean problema, pero el caso de enfermedad es más difícil de resolver: basándose en la calidad de los otorgantes, Claude LARQUIE opta por sumar estos casos al total de los firmantes. Yo estoy mucho más reservado. En unos pocos casos, el escribano puede autorizar esta equivalencia especificando «aunque sabe firmar, no lo hizo por...». Pero, en los demás casos, icómo estar seguro de que la viuda de un letrado o de un maestro artesano sabia firmar? La experiencia enseña que, en tales casos, las dos posibilidades existen. Y ¿cómo resolver los casos (que son los más numerosos) en que la calidad o la profesión del otorgante no aparece (a menos de encontrar, por casuali- 
dad, otra acta del mismo individuo en otro protocolo)? ante esas dificultades, hemos optado provisionalmente por clasificar aparte a los impedidos de firmar.

En cuanto a las actas que están firmadas, no nos hemos contentado con una sencilla separación entre firmantes y no firmantes: creemos que el estudio de la firma puede matizarse y permitir diferenciar niveles de firma:

-hay, por ejemplo, esas firmas dibujadas torpemente letra tras letra, de que hablaba Yves CASTAN. Tal tipo de firma, que recuerda la escritura de un nifoo en el primer año del colegio, es relativamente frecuente en las actas, y constituirá uno de nuestros niveles.

-hay, por lo contrario, las firmas adornadas con una rúbrica, frecuentes entre los letrados y los que, por su actividad, tenían una práctica diaria de la escritura.

Estas consideraciones nos han llevado a adoptar la clasificación en cuatro niveles propuesta por BENNASSAR, añadiendo un quinto nivel para los impedidos, o sea:

-nivel A (firma bien): la firma revela, por su facilidad, su rapidez, una práctica segura de la escritura. En esta categoría clasificaremos las firmas adornadas de ciertos letrados y las, sencillas pero firmes, de comerciantes o funcionarios. Este primer grupo reunirá pues el nivel culto de los letrados y el nivel práctico de los que escriben diariamente.

-nivel B (firma): es el nivel rudimentario de los que logran escribir su apellido, pero sin la soltura, la firmeza de los de la primera categoría, por falta de habilidad o de práctica.

-nivel $€$ (firma mal): son esas firmas torpes, dibujadas letra tras letra, con las letras separadas a menudo. Los individuos que integran este grupo no serán totalmente analfabetos, pero tendrán un nivel cultural muy bajo.

-nivel D (no sabe firmar): la incapacidad para firmar, atestiguada por el escribano, significa para nosotros el analfabetismo total. firmar.

- nivel E (no puede firmar): es el grupo de los enfermos, impedidos de

Son esos cinco niveles los que hemos tratado de diferenciar en nuestras encuestas llevadas a cabo en Santander, León, Burgos, Barcelona, Madrid, Ciudad Real, Murcia y Zafra. Para dar mayor coherencia a la investigación, y cierta dinámica a la apreciación de los resultados, hemos optado por concentrar nuestros análisis en dos períodos del siglo XVIII: los años de 1750-1755 y los últimos años del siglo, aunque el período estudiado puede variar según las ciudades y provincias (un año sólo en Madrid, cinco años o más en ciudades medias o pequeñas).

\section{Los resultados}

\subsection{Resultados globales}

Teniendo en cuenta los resultados de once encuestas llevadas a cabo en ocho ciudades o provincias distintas entre 1750 y 1805 , y que analizan 9.873 firmas, 
llegamos a los resultados siguientes:

$\begin{array}{rr}\text { Nivel A } & 14,22 \% \\ \text { B } & 17,73 \% \\ \text { C } & 11,69 \% \\ \text { D } & 50,04 \% \\ \text { E } & 6,30 \%\end{array}$

Dada la poca importancia cuantitativa del último grupo, y deseoso de evitar cualquier extrapolación imprudente, he optado por eliminar esos 622 casos, trabajando sobre los 9.251 casos bien especificados, que dan las proporciones siguientes:
nivel A (firma bien)
B (firma)
$15,17 \%$
C (firma mal)
$18,92 \%$
o sea un $34 \%$ de alfabetizados
D (no sabe firmar
$12,48 \%$ de semianalfabetos
$53,41 \%$ de analfabetos totales.

Estamos lejos de los resultados eufóricos de BENNASSAR, a partir de las fuentes inquisitoriales (un $75 \%$ de alfabetizados). Y aún debemos tener en cuenta el hecho de que nuestra muestra privilegia las clases sociales más favorecidas, o sea que nuestros resultados son demasiado optimistas.

Para Madrid en 1650, Claude LARQUIE proponía una corrección de un $10 \%$ para compensar la menor representación de las clases populares. Este porcentaje me parece pertinente para una ciudad en que las declaraciones de pobres eran numerosas, pero es insuficiente para las demás provincias en que este tipo de documento no existía. Propongo pues aplicar dos coeficientes de corrección distintos según las zonas:

-una tasa del $10 \%$ para Madrid,

- una tasa del $25 \%$ para las demás regiones, que corresponderán a la ausencia en nuestra muestra de los representantes de las clases inferiores, que sumaremos al nivel D de los analfabetos.

Después de esta corrección, llegamos al resultado siguiente:
nivel A (firma bien)
nivel $B$ (firma) $12,54 \%$
nivel C (firma mal)
$15,51 \%$
o sea un $28,4 \%$ de alfabetizados
nivel $D$ (no sabe firmar)
$10,20 \%$ de semianalfabetos

Estas tasas no pretenden tener ninguna exactitud científica: se trata de una evaluación aproximada de-la alfabetización en España en la segunda mitad del XVIII, que trataremos de precisar marcando la evolución cronológica, las diferencias por regiones, por sexos y por lugares de residencia.

\subsection{Evolución cronológica}

A mediados de siglo (entre 1750 y 1759), disponemos de los resultados de cinco encuestas realizadas en Santander (331 casos), Burgos (1.323), Madrid (1.007), Ciudad Real (566) y Murcia (1.657), o sea 4.884 firmas. El resultado global, en tasas corregidas es el siguiente: 


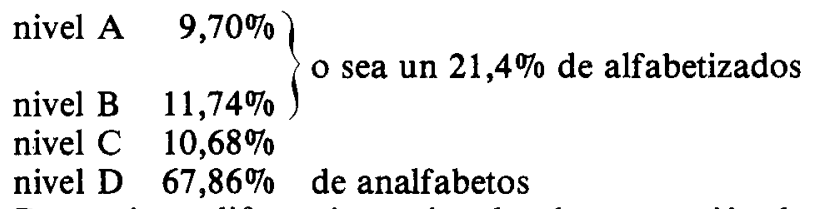

Pero existen diferencias regionales: la proporción de alfabetizados alcanza un $32,23 \%$ en Madrid un $25,62 \%$ en Burgos un $14,46 \%$ en Murcia un $12,89 \%$ en Ciudad Real.

Los analfabetos completos representan aún

el $51,38 \%$ de la población madrileña,

el $68,25 \%$ de la población de Santander,

el $70,89 \%$ de la población de Murcia,

el $81,87 \%$ de la población de Ciudad Real.

A fines del XVIII (entre 1787 y 1805), disponemos de ocho encuestas en Santander (311 casos), León (587), Burgos (1.482), Barcelona (304), Madrid (997), Ciudad Real (375), Murcia (345) y Zafra (597), o sea 4.998 firmas.

El resultado global en tasas corregidas es el siguiente:

nivel A $15,07 \%$ ?

nivel B $19,15 \%$ o sea un $34,2 \%$ de alfabetizados

nivel C $\quad 9,79 \%$

nivel D 55,97\% de analfabetos completos,

resultado que podemos comparar con el $37 \%$ de firmantes en Francia en 17861790, según la encuesta de MAGGIOLO. El progreso de la alfabetización durante la segunda mitad del siglo (época de las Luces) alcanza así un 13\%, pero este beneficio es muy variable según las regiones: apenas un $2 \%$ en Santander $(27,2 \%)$,

pero un $8 \%$ en Ciudad Real $(20,4 \%)$,

un $10 \%$ en Madrid $(42,7 \%)$,

y un $16 \%$ en Murcia $(30,6 \%)$.

El analfabetismo completo queda reducido a un $74 \%$ de la población en Ciudad Real, un $65 \%$ de la población en Santander, un $64 \%$ de la población en Murcia, un $56 \%$ de la población en Burgos, un $48 \%$ de la población en Madrid.

Notaremos que la tasa global de analfabetismo a fines del siglo XVIII en nuestra encuesta $(55,97 \%)$ es ya un poco inferior a la registrada en el censo de 1900: $56,07 \%$. Si tenemos en cuenta el hecho que la alfabetización seguirá adelantando en los primeros decenios del XIX, eso supone una regresión de la alfabetización en la segunda mitad del XIX, probablemente a raíz de la desamortización, hipótesis ya adelantada por BENNASSAR. Es un punto que tendrá que ser confirmado por los desarrollos ulteriores de nuestra encuesta. 
Si tratamos de precisar el análisis de nuestros resultados, dos parámetros se imponen: el sexo y el lugar de residencia.

\subsection{Alfabetización masculina y femenina}

No es ninguna sorpresa comprobar que, en el siglo XVIII, la alfabetización sigue siendo un fenómeno mayoritariamente masculino. A mediados del siglo, saben firmar (niveles A y B) el 30,02\% de los hombres y el 4,67\% de las mujeres. A finales del siglo, el $42,90 \%$ de los hombres están alfabetizados, contra el $13,46 \%$ de las mujeres, o sea que, durante la segunda mitad del siglo, el progreso ha alcanzado un $12,8 \%$ para los hombres y un $8,7 \%$ para las mujeres: la diferencia entre alfabetización masculina y femenina pasa así de un $25 \%$ en 1750 a un $29 \%$ en 1800 .

Si comparamos estos resultados con las medidas francesas sacadas de la encuesta de MAGGIOLO, nos damos cuenta de que la tasa masculina española es un poco inferior a la francesa, pero honorable (un $42,9 \%$ contra un $47 \%$ ); en cambio, la tasa femenina representa menos de la mitad de lo que es en Francia: un $13,46 \%$ contra un $27 \%$. El retraso de la alfabetización en España se debe ante todo al nivel cultural muy bajo de las mujeres.

\subsection{Alfabetización urbana y rural}

Otro parámetro esencial: las ciudades están más alfabetizadas que el campo. Tanto en 1750 como en 1797 , el mejor resultado alcanzado en nuestras encuestas es el de Madrid:

-en 1750, un $32,23 \%$ de alfabetizados (niveles A y B) contra un $16,36 \%$ de semianalfabetos (nivel C) y un $51,38 \%$ de analfabetos completos (nivel D);

-en 1797 , un $42,77 \%$ de alfabetizados, contra un $9,10 \%$ de semianalfabetos y un $48,10 \%$ de analfabetos completos.

Si sumamos los grupos A, B y C, nos damos cuenta de que, a fines del XVIII, casi el $53 \%$ de los madrileños son capaces de firmar, bien o mal, su testamento (el $68 \%$ de los hombres y el $30 \%$ de las mujeres). Estamos lejos, sin embargo, de los resultados de París, donde en vísperas de la Revolución, el $90 \%$ de los hombres y el $80 \%$ de las mujeres sabían firmar. ${ }^{(13)}$

Nuestras encuestas nos permiten además comparar el nivel de alfabetización en la ciudad y en el campo en una misma provincia. Por ejemplo, en Burgos en 1750-1755:

$\begin{array}{rccc} & \text { ciudad } & \text { campo } & \text { diferencia } \\ \text { alfabetizados (niveles A y B): } & 28,05 \% & 8,21 \% & 20,00 \% \\ \text { (nivel D): } & 64,07 \% & 86,07 \% & 22,00 \% .\end{array}$

En 1795-1799, tendremos:

$$
\text { (nivel D): } 64,07 \% \quad 86,07 \% \quad 22,00 \% \text {. }
$$

$$
\begin{array}{rlll}
\text { alfabetizados } & 34,05 \% & 11,07 \% & 22,08 \% \\
\text { analfabetos } & 52,01 \% & 80,08 \% & 28,07 \% \text {. }
\end{array}
$$

O sea que la diferencia entre campo y ciudad se ha agravado aun en la segunda mitad del siglo (las escuelas de primeras letras se fundan en las zonas urbanas antes que en las zonas rurales). Una vez más, esta diferencia se debe en gran parte 
a las tasas muy bajas de alfabetización femenina en el campo:

en Burgos, por ejemplo, ninguna aldeana está alfabetizada (nivel A o B) en 1750 y 1795

en Murcia, en 1750, las tasas de alfabetización en la huerta alcanzan un 9\% para los hombres contra un $0,4 \%$ para las mujeres.

Varias encuestas llevadas a cabo en Francia han mostrado que la residencia en la ciudad tiende a reducir la diferencia entre alfabetización masculina y femenina (por ejemplo, el $90 \%$ y el $\mathbf{8 0 \%}$ en París, lo que Daniel ROCHE explica por los oficios urbanos y por las constantes incitaciones a la lectura práctica y utilitaria que provoca la calle de una gran ciudad: rótulos, carteles, etc...). En España, la residencia en la ciudad, lejos de reducir la diferencia entre alfabetización masculina y femenina, la aumenta:

en Burgos, en 1750, el 14,5\% de los hombres y el $0 \%$ de las mujeres están alfabetizados en el campo (diferencia 14,5), contra respectivamente el $41,6 \%$ y el $10,3 \%$ en la ciudad (diferencia 31,3 );

en Murcia, en 1750, el 8,98\% de los hombres y el 0,4\% de las mujeres están alfabetizados en el campo (diferencia 8,5 ) contra respectivamente el $28,33 \%$ y el $4,3 \%$ en la ciudad (diferencia 24 ).

O sea que si la ciudad favorece indudablemente la promoción masculina por los oficios que el hombre desempeñará en ella, no parece tener efectos tan sensibles sobre la promoción femenina hasta fines del siglo XVIII. Este punto tendrá que ser confirmado, pero estamos aquí ante un problema de sociedad que será interesante profundizar, y que tiene que ver a la vez con el papel económico y social de la mujer, y con las mentalidades.

\subsection{Alfabetización y grupos socioprofesionales}

La calidad o profesión del otorgante no está siempre especificada en los protocolos, pero la masa de documentos de que disponemos es suficiente para permitirnos adelantar conclusiones ya bastante seguras, a un nivel muy general.

La nobleza tiene un nivel de alfabetización bastante elevado, pero con diferencias regionales sensibles entre las provincias del norte, con una nobleza numerosa y heterogénea, y las del sur, con una nobleza menos numerosa, pero más homogénea y más alfabetizada. En León, a fines del XVIII, si todos los hombres nobles saben firmar, bien o mal (niveles A y B: $72,7 \%$, nivel C: $27,2 \%$ ), la tercera parte de las mujeres son analfabetas: niveles $A$ y B: $36,2 \%$, nivel C: $23,1 \%$, nivel D: $31,5 \%$.

En Zafra, pequeña ciudad rica de Extremadura, capital del ducado de Feria, el $90 \%$ de los hombres y el $70 \%$ de las mujeres nobles saben leer y escribir (niveles A y B), los demás firman mal (nivel C) en los últimos años del XVIII.

Los letrados constituyen el grupo socioprofesional que tiene el nivel de alfabetización más elevado: generalmente, un $70 \%$ del nivel A y un $30 \%$ del nivel B.

Los militares tienen un nivel de alfabetización superior a la media general de los hombres, pero dependiente, sin embargo, de su situación en la jerarquía. Todos los oficiales y suboficiales saben firmar, y los alfabetizados son bastante numerosos entre los soldados rasos: por ejemplo, hemos encontrado en Madrid 
en 1797 cinco guardias de corps que firman bien (nivel A), contra un soldado inválido analfabeto, y contra tres soldados de origen rural analfabetos en León.

El clero: el clero secular está totalmente alfabetizado (nivel A o B), pero no es el caso del clero regular: las renuncias de novicios o de religiosas, relativamente frecuentes en los protocolos, ponen de relieve esta diferencia:

-en Ciudad Real, todos los presbíteros firman A o B, pero de 13 monjas, una firma $\mathrm{B}$, las demás $\mathrm{C}$;

-en León, donde todos los presbiteros también firman A ○ B, siete renuncias de religiosas dan los resultados siguientes: $3 \mathrm{~B}, 3 \mathrm{C}, 1 \mathrm{D}$. Las tres novicias que firman B son de familia noble, hijas de D. Francisco Arnaiz de las Revillas, Caballero de Santiago, que paga 22.000 reales en monedas de oro al contado (cuando la costumbre era pagar sólo una parte de la dote al firmar la renuncia). El paralelismo entre nivel económico y nivel de alfabetización es aquí evidente: las firmas B corresponden a dotes de $\mathbf{2 2 . 0 0 0}$ reales, las firmas $\mathrm{C}$ a dotes de 17 a 15.000 reales, y la firma $\mathrm{D}$ a una ausencia de dote. (14)

Los comerciantes: en Madrid (1797), hemos encontrado un grupo de 41 comerciantes, entre los cuales 16 firman A, 16 firman $\mathrm{C}$ y 6 no saben firmar.Firman bien varios miembros de los Cinco Gremios Mayores, un librero. Entre los que no saben firmar, un carbonero.

Ciertos documentos permiten tener una idea bastante precisa del nivel de alfabetización dentro de un mismo sector de actividad:

-17 hombres participan en una reunión del gremio de hosteleros con el fin de nombrar oficiales (Madrid, 6 de diciembre de 1797): uno firma A, 11 firman B, 1 C y 4 D;

-doce hombres y dos mujeres participan en una reunión del gremio de Mercaderes de Hierro con el fin de otorgar un poder general a sus directores (Madrid, 1797): diez hombres firman A, dos firman B, mientras las dos mujeres firman C y D.

Un ejemplo más del retraso de la alfabetización femenina, hasta cuando hombres y mujeres desempeñan una misma función económica.

El artesanado: en el mundo complejo de las artes y oficios, es difícil trazar la frontera entre alfabetizados y no alfabetizados. El nivel de alfabetización depende a la vez del oficio, de la riqueza y de la cualificación (los maestros están más alfabetizados que los oficiales). Hay oficios en que saber firmar es prácticamente una regla: los plateros, pintores y doradores, vidrieros; hay oficios en que saber firmar es una excepción: curtidor, arriero, panadero, etc... Pero, en la mayoría de los casos, coexisten firmantes y no firmantes.

Una encuesta llevada a cabo en Guadalajara a mediados del siglo XVIII revela que, en la Real Fábrica de Paños, las tasas de analfabetismo eran las siguientes en los distintos sectores de actividad:

-desmontadoras (mujeres exclusivamente)

$100 \%$

-emborradores

$64 \%$

- estambreros

$56 \%$

-tundidores

$48 \%$

- perchadores

$45 \%$

- tejedores de paños

$38 \%$

- tejedores de sarguetas

$33 \%$. ${ }^{(15)}$ 
Habrá que multiplicar las encuestas para apreciar las situaciones locales peculiares, las diferencias regionales. Pero los resultados obtenidos son a veces difíciles de explicar: en Madrid (1797), el 36\% de las 426 declaraciones de pobres otorgadas ante los escribanos están firmadas:

$\begin{array}{lr}\text { nivel A } & 3,52 \% \\ \text { nivel B } & 24,17 \% \\ \text { nivel C } & 8,45 \%\end{array}$

Entre los firmantes, varios presbíteros, un maestro cirujano, un platero y muchos pobres de solemnidad. ¿Son todos ellos verdaderamente pobres? ¿Quiénes son esos pobres de solemnidad? ¿Cómo aprendieron a escribir? El mundo de las clases populares, tan complejo y contradictorio, sigue siendo el más difícil de siluetear.

\subsection{Firma y matrimonio}

Los testamentos (o declaraciones de pobres) otorgados por parejas permiten estudiar la alfabetización dentro del matrimonio. Tomaremos el ejemplo de Murcia en 1750 ( 259 parejas): $(20 \%)$,

-caso 1: los dos cónyuges saben firmar, bien o mal (A, B, C): 52 casos

-caso 2: sólo el hombre sabe firmar, bien o mal: 122 casos $(47,1 \%)$,

- caso 3: sólo la mujer sabe firmar bien o mal: 2 casos $(0,7 \%)$,

- caso 4: ninguno de los dos sabe firmar (D): 83 casos (32\%).

A pesar del desnivel inevitable entre hombres y mujeres, la tasa de alfabetización en los matrimonios es superior a la tasa registrada para cada sexo: el 20\% de las mujeres casadas firman, bien o mal, contra el $11,4 \%$ de las mujeres en general.

Algunos ejemplos de matrimonios en que los dos cónyuges firman bien (nivel A) en Madrid (1797): nobles, letrados, comerciantes (miembros de los Gremios Mayores) y un criado (arriero del Señor Infante Don Antonio).

Matrimonios en que el marido firma bien (A) y la mujer no sabe firmar (D): un abogado de los Reales Consejos, un maestro vidriero, un guardia de corps.

Vemos que las reglas son difíciles de dictar, pero las parejas de nobles son las que suelen tener una mayor armonía cultural.

\section{Conclusion}

El balance presentado al cabo de cinco años de investigación revela unos resultados positivos:

-al nivel metodológico, con la definición de un método de investigación, que estamos tratando de hacer más eficaz gracias a la utilización de la informática,

-al nivel cuantitativo, con casi diez mil firmas estudiadas en ocho provincias distintas, que nos permiten definir ya una problemática y adelantar unas primeras conclusiones.

Nuestras encuestas se prolongan en varias áreas ya exploradas, donde trata- 
mos de profundizar y matizar los primeros resultados alcanzados; otras encuestas nuevas empiezan en Zaragoza, Salamanca y Palencia. Nuestra intención es prolongar la labor iniciada, extendiérdola a las zonas en que todavía, por razones materiales, no hemos podido empezar a trabajar (en particular, Andalucía, el área valenciana y Galicia), para llegar a las veinte mil firmas, que es el objetivo que nos habíamos fijado al principio.

Pero la medida cuantitativa de la alfabetización es sólo el segundo nivel de este inmenso campo de investigación que hay que sobrepasar. Los archivos notariales nos ofrecen amplia materia con los inventarios de bienes para abordar el tercer nivel de estudio: el de la difusión del libro y de los lectores. Los inventarios de bienes de los libreros que nos permiten saber qué libros se vendian (se leían) en una ciudad, y los inventarios de bibliotecas, que nos permiten saber, a nivel cualitativo, quiénes poseían libros y qué clases de libros, constituyen las bases para una posible historia social de la cultura en el siglo XVIII español.

El programa es ambicioso, y hasta podrá parecer utópico. Pero creo que, si logramos unir nuestros esfuerzos a los de los equipos de investigadores que trabajan en diferentes universidades españolas, podemos esperar adelantar más rápidamente y mejor hacia estas perspectivas.

\section{NOTAS}

(1) Aunque este tipo de estudio se está desarrollando en España: además de los trabajos de J. Eloy GELABERT en Galicia, hemos descubierto con mucho interés el programa de investigación de Antonio VIÑAO FRAGO y su equipo en Murcia, con quien esperamos establecer una fructuosa colaboración.

(2) Véase el artículo de Carmen LABRADOR HERRAIZ sobre los maestros de primeras letras en la Alcarria en Actas del segundo Simposio sobre el Padre Feijoo y su siglo, t. II, Oviedo, 1983.

(3) Un documento de fundación de una escuela de primeras letras en León a fines del XVIII precisa así el salario del maestro:

«podrá llevar un real de cada uno al mes, siendo de los que sólo aprenden a leer, y dos reales si aprendieren a escribir, y lo mismo si a escribir y a contar». (León, Archivo Histórico de Protocolos, citado por Jean-Marc BUIGUES, Mémoire de D.E.A., Montpellier, 1982.

(4) LUZURIAGA: El analfabetismo en España, Madrid, 1919.

(5) Las tres posiciones analizadas están expuestas en:

Yves CASTAN: Honnêteté et relations sociales en Languedoc, París, Plon, 1974.

Jean MEYER: «Alphabétisation, lecture et écriture», Actes du 95ème Congrès des Sociétés Savantes, t. 1, París, 1974 (p. 331 y sig.).

François FURET et Wladimir SACHS: «La croissance de l'alphabétisation en France (XVIII ${ }^{\text {ème_ }}$ XIX ${ }^{\text {ème }}$ siècles)", Annales E.S.C., mai-juin 1974, p. 714-735.

Sobre este punto, véase también P. CHARTIER, D. JULIA et M. COMPERE: L'éducation en France du XVI ème au XVIII ème siècle, Paris, SEDES, 1976, en particular el capítulo $1^{\circ}$, cuya argumentación seguimos.

(6) Juan Eloy GELABERT: «Lectura y escritura en una ciudad provinciana del siglo XVI: Santiago de Compostela», Bulletin Hispanique, 1982.

(7) Jeannette VILLA ha empezado a trabajar sobre el donativo de 1797-1799 en Murcia. Por otra parte, Jean-Marc BUIGUES analiza en León los «padrones de la moneda forera» entre 1578 y 1816. 
(8) Marie-Christine RODRIGUEZ et Bartolome BENNASSAR: «Signatures et niveau culturel des témoins et accusés dans les procès d'Inquisition du ressort du tribunal de Tolède (1525-1817) et du ressort du tribunal de Cordoue (1595-1632)", Caravelle, n 31, 1978, P. 17 y sig.

(9) Claude LARQUIE: «La alfabetización de los madrileños en 1650», Anales del Instituto de Estudios Madrileños, t. XVII, 1980, p. 223-252 (traducción francesa en Revue d'histoire moderne et contemporaine, 1981).

(10) Los resultados presentados se basan en mis propias investigaciones en Madrid (año de 1797, resultados parciales todavía), y en las encuestas llevadas a cabo por los siguientes estudiantes y doctorandos bajo mi dirección: Jean-Marc BUIGUES (León), Erondine RODRIGUEZ (Santander), Magali PANSIER (Burgos), Mercedes GARAYOA (Barcelona), Sylvie IMPARATO (Madrid, 1750), Marie-Paule ROMERA (Ciudad Real), Frédérique BAPTISTE (Murcia, 1750), Jeannette VILLA (Murcia, 1797), Françoise POURRET (Zafra).

(11) La inexistencia de las «declaraciones de pobres» fuera de Madrid se debe a la situación jurídica peculiar de la Corte. A fines del XVIII, las «declaraciones de pobres» son el documento más frecuente en los protocolos madrileños. Si la mayoria de los otorgantes parecen ser «verdaderos pobres», algunas son sospechosas (véase mi tesis doctoral: Paupérisme et rapports sociaux à Madrid au XVIII ${ }^{e m e}$ siècle, Lille, 1978,2 t., en part. cap. II, traducción española en la revista Estudios de Historia Social, Madrid, $\mathrm{n}^{\circ}$ I-II, 1980 y $\mathrm{n}^{\circ}$ I-II, 1982).

(12) Utilizaremos estos datos para un estudio en preparación sobre la inmigración en Madrid a fines del siglo XVIII.

(13) Daniel ROCHE: Le Peuple de París. Essai sur la culture populaire au XVIII'me siècle Paris, Aubier-Montaigne, 1981 .

(14) J. M. BUIGUES: Memoria de D.E.A. ya citada. 1984.

(15) Vincent PARELLO: Guadalajara en el siglo XVIII, mémoire de mâ̂trise, Montpellier, 